\title{
Automated discrimination of psychotropic drugs in mice via computer vision-based analysis
}

\author{
Zeynep Yucel ${ }^{\mathrm{a}}$, Yildirim Sara ${ }^{\mathrm{c}, *}$, Pinar Duygulu $^{\mathrm{b}}$, Rustu Onur ${ }^{\mathrm{c}}$, Emre Esen $^{\mathrm{c}}$, A. Bulent Ozguler ${ }^{\mathrm{a}}$ \\ a Department of Electrical and Electronics Engineering, Bilkent University, Ankara, Turkey \\ ${ }^{\mathrm{b}}$ Department of Computer Engineering, Bilkent University, Ankara, Turkey

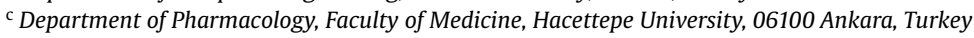

\section{A R T I C L E I N F O}

\section{Article history:}

Received 16 February 2009

Received in revised form 12 March 2009

Accepted 17 March 2009

\section{Keywords:}

Computerized video analysis

Drug discrimination

Locomotor activity

Open field

Automatization

\begin{abstract}
A B S T R A C T
We developed an inexpensive computer vision-based method utilizing an algorithm which differentiates drug-induced behavioral alterations. The mice were observed in an open-field arena and their activity was recorded for $100 \mathrm{~min}$. For each animal the first $50 \mathrm{~min}$ of observation were regarded as the drug-free period. Each animal was exposed to only one drug and they were injected (i.p.) with either amphetamine or cocaine as the stimulant drugs or morphine or diazepam as the inhibitory agents. The software divided the arena into virtual grids and calculated the number of visits (sojourn counts) to the grids and instantaneous speeds within these grids by analyzing video data. These spatial distributions of sojourn counts and instantaneous speeds were used to construct feature vectors which were fed to the classifier algorithms for the final step of matching the animals and the drugs. The software decided which of the animals were drug-treated at a rate of $96 \%$. The algorithm achieved $92 \%$ accuracy in sorting the data according to the increased or decreased activity and then determined which drug was delivered. The method differentiated the type of psychostimulant or inhibitory drugs with a success ratio of $70 \%$ and $80 \%$, respectively. This method provides a new way to automatically evaluate and classify drug-induced behaviors in mice. Crown Copyright (c) 2009 Published by Elsevier B.V. All rights reserved.
\end{abstract}

\section{Introduction}

Behavioral studies in biological research are mostly based on the observation and evaluation of motor activity of animals in experimental models. Recording locomotion in the open field or an arena is widely used to investigate the behavioral alterations of the animals in response to therapeutic interventions, genetic mutations and for evaluation of behavioral responses to psychoactive drugs. A variety of methods are available to measure motor activity. Conventional and widely used photobeam apparatus monitors horizontal and vertical locomotor activity, area entries, and the occurrence of different activities, such as rearing. The system generates a signal when an animal interrupts the infrared light and suitable arrangement of sensors register movements in the desired direction. The standard photobeam apparatus has been used for recording motor activity for preclinical drug evaluation (Beninger et al., 1985; Clarke et al., 1985; Teicher et al., 1996; Robles, 1990). Some drawbacks of this system were eliminated by continuous-wave Doppler radar (CWDR) as an alternative to the standard photobeam box (Pasquali and Renzi, 2005). Multilayer feed-forward neural networks, which are fed with the power spectrum estimation and Root

\footnotetext{
* Corresponding author. Tel.: +90 312 3051086; fax: +90 3123105312 .

E-mail address: ysara@hacettepe.edu.tr (Y. Sara).
}

Mean Square (RMS) values of these signals, helped them to classify the behavior as exploring, grooming and sedation. Drai et al. (2000), using data measured by a standard photobeam tracking system introduced an algorithm that segments rodent locomotor behavior and demonstrated the effects of amphetamine and phencyclidine in rats. Other than force sensors, infrared photobeam recording and CWDR, video capturing has been used in tracking rodent motion in behavioral studies (Noldus et al., 2001; Vorhees et al., 1992).

Computer systems utilizing suitable software are employed to analyze digital video recordings of the activity of experimental animals to evaluate their behavior. Automated observation with video capturing presents significant advantages over previous methods. In these methods, animal behavior is recorded more reliably because the computer algorithm is not subjective, and it is not prone to operator bias. In contrast to visual observation, video tracking may also perform pattern analysis on a video image of the observed animal and derive quantitative measurements of the behavior (Noldus et al., 2001). Automated observation using video tracking is particularly suitable for recording locomotor activity. Activity is expressed as spatial measurements of distance travelled, speed, and acceleration (Buresova et al., 1986; Dielenberg et al., 2006; Spruijt and Gispen, 1983; Spruijt et al., 1998). In a recent study Shih and Young (2007) reported a combination of an accelerometer and video camera system to simultaneously measure vibration and 
locomotion activity and compared the effects of amphetamine and pentobarbital.

Discrimination of variations in the locomotor activity is important in behavioral studies. A system which is capable of detecting behavioral alterations in response to pharmacological manipulations could prove very useful in behavioral and neuropharmacological studies, as well as in drug screening and toxicology studies. Thus, the present study was conducted to develop an automated system for recording and analyzing the locomotor activity of mice in response to pharmacological manipulation. We present here a video tracking method which utilizes an algorithm to detect and discriminate drug responses elicited by diverse pharmacological groups. In order to evaluate the method employed we tested typical pharmacological agents with well-described behavioral effects. We used amphetamine and cocaine as the stimulant drugs and diazepam and morphine as the inhibitory agents. Our main contribution lies in the construction of the feature vectors which represent the behavior of the subject in such a way that the behavioral distinctions are preserved and displayed clearly.

\section{Materials, methods, and results}

\subsection{Animals}

Male albino mice weighing 30-35g were used in these experiments. Mice were housed in groups of three per cages in a temperature-controlled room $\left(23 \pm 1^{\circ} \mathrm{C}\right)$ with a relatively humidity of $45-70 \%$ and kept in a $12 \mathrm{~h}: 12 \mathrm{~h}$ light/dark cycle (illuminated between 1800 and $0600 \mathrm{~h}$ ). Access to food and water was unrestricted. The methods and procedures of the present study were approved by the ethics committee of Hacettepe University (2008/71-4).

\subsection{Drugs}

D-Amphetamine hydrochloride and diazepam were obtained from Sigma Chemical Co. (USA), whilst cocaine hydrochloride and morphine hydrochloride were obtained from Etablissements Roques, France and Verenigde Pharmazeutische Fabriken, Holland, respectively. All drugs were dissolved in saline.

\subsection{Open field measurements}

Mice were taken one at a time from their standard home cages, weighed and marked. Then animals were transferred to the open field apparatus and their video images were recorded as they explored. The open field consisted of a square base, $45 \mathrm{~cm} \times 45 \mathrm{~cm}$ with glass walls $45 \mathrm{~cm}$ high. The floor of the arena was painted matt black, and the arena was illuminated by means of an incandescent lamp of $40 \mathrm{~W}$, positioned above the base providing a homogeneous illumination in the arena. The arena was located in a dark room and it is kept away from odor or sound. An adjustable surveillance camera (Fly WC-OML300, China) was positioned $60 \mathrm{~cm}$ above the base of the arena and was connected to a personal computer. The behaviors of the mice were recorded at a frame rate of $10 \mathrm{~Hz}$.

In this study, 29 animals were divided into five groups. For each animal, video images were recorded in two following sessions. In the first session, baseline activity of the mice was recorded for 50 min without drug administration. Immediately after this session, animals received an injection of cocaine, amphetamine, diazepam, or morphine and were placed back into the arena for another $50 \mathrm{~min}$. Each animal was used only once for each drug. All injections were given intraperitoneally (i.p.) in a volume of $10 \mathrm{ml} / \mathrm{kg}$. All the drugs were dissolved in saline and were administered at doses of $10 \mathrm{mg} / \mathrm{kg}$ for cocaine $(n=6), 10 \mathrm{mg} / \mathrm{kg}$ amphetamine $(n=6) 10 \mathrm{mg} / \mathrm{kg}$ morphine $(n=6)$ and $10 \mathrm{mg} / \mathrm{kg}$ diazepam $(n=6)$. The initial $10 \mathrm{~min}$ of each session were discarded. During this period animals resumed their baseline locomotor activity following manipulation.

\subsection{Statistical analysis}

Within group comparisons among baseline and post-treatment activities were made using two-way ANOVA. $P$ value of less than 0.05 was considered statistically significant.

\subsection{Data analysis}

Data analysis method composed of mainly two stages: (i) behavior representation step, which includes motion tracking and feature extraction, and (ii) classification step, where the videos are labeled according to the animals behavioral differences.

\subsubsection{Drug-induced alteration in locomotion}

Prior to the evaluation of psychotropic drug effects we studied the effects of saline injection. Six separate mice injected with saline did not display altered locomotor activity and their cumulative travelled distance curves overlapped before (baseline) and after the injections (Fig. 1A inset). Then we compared the effects of drugs with their untreated (baseline) activity. Examination of the video recordings and cumulative travelled distances (Fig. 1) revealed that amphetamine $(P<0.006)$ and cocaine $(P<0.03)$ increased locomotor activity compared to the pre-drug control period, while morphine $(P<0.01)$ and diazepam $(P<0.04)$ inhibited locomotion. However, amphetamine- and cocaine-induced increased locomotor activity exhibited different characteristics. Following administration of both amphetamine and cocaine the animals displayed accelerated movement and the distance they travelled significantly increased with respect to the controls (Fig. 1A). Amphetamineadministered animals preferentially moved along the edges of the arena, while cocaine-treated animals moved throughout the arena including the central reagents, displaying a motion of more distributed nature (Figs. 2 and 3). Morphine and diazepam inhibited locomotion (Fig. 1B), however this inhibition also displayed different characteristics (Figs. 2 and 3). Under the influence of morphine, the animals mostly remained sedated in one restricted area, generally located near the corners of the arena. Diazepam-treated animals also remained sedated but to a lesser extent and they appeared slightly more active around the edges of the arena, with respect to the morphine group.

Based on these observations, we developed a hierarchical scheme to differentiate the administered drugs by analyzing the video of the mouse under the influence of a given drug. The structure of the hierarchical classification (HC) scheme is illustrated in Fig. 4A. In Step 1, it is investigated whether the animal is exposed to drugs used in this study or it exhibits a drug-naïve behavior. If the mouse is detected to be drug-naïve, no further investigation is performed. If the animals' activity is different from drug-naïve condition, then the animal is considered to be drug-treated. In Step 2 , the data is classified as increased or decreased, according to the activity of the mouse. Finally at Step 3 the activity was analyzed separately depending on its type and the final decision was reached considering the previously acquired drug characteristics.

Behavioral analysis is composed of motion tracking and feature extraction steps. In the motion tracking step, the location of the animal is determined at each video frame. Feature extraction step uses this information in two stages to represent the area explored by the animal and the speed of the motion which provides distinctive characteristics for behavioral analysis.

\subsubsection{Motion tracking algorithm}

In order to track the motion of the animal, a video of $N$ frames is recorded, with each video frame $F_{n}, 1 \leq n \leq N$, consisting of $M \times M$ pixels. The path followed by the animal is found by positioning the 

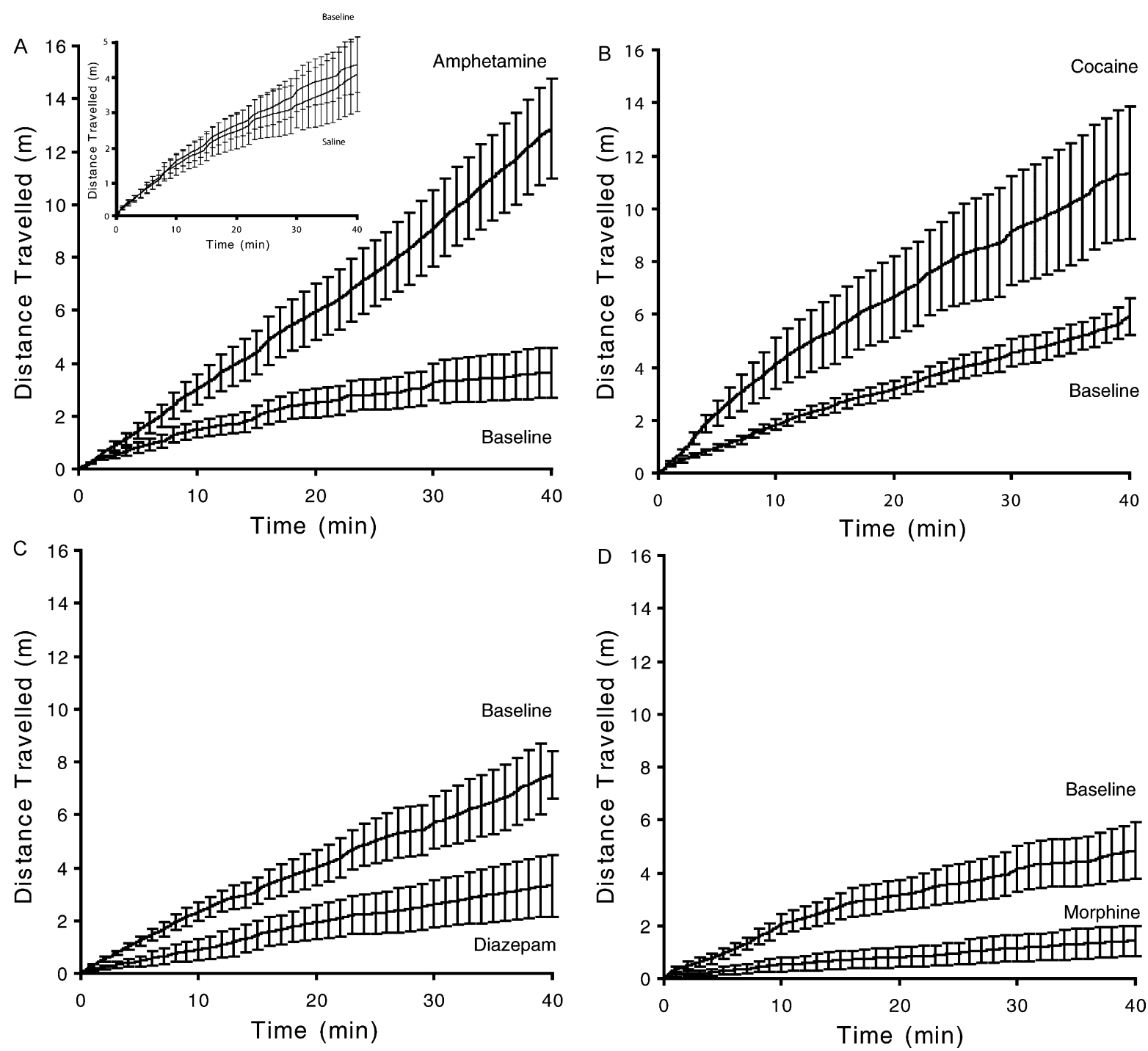

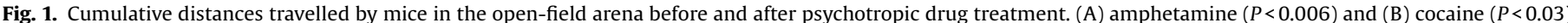

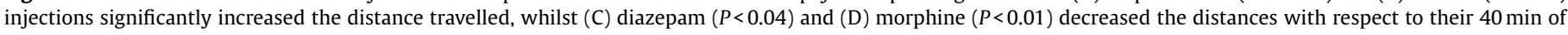

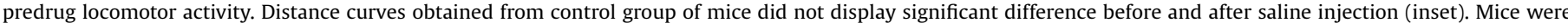

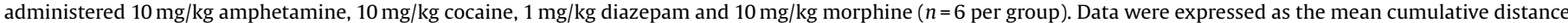
travelled \pm SEM over the 40 min of test period.

location of the subject in each frame. For this purpose, first, a background model, which we refer as $B G$, is constructed by recording an image of the empty arena before the animal is placed. The location in each frame is then found by subtracting the background model $B G$, from each frame $F_{n}$ and then marking the pixels different from the background. This process first requires the construction of a difference image $D_{n}$ obtained by subtracting the background model $B G$ from $F_{n}$ for each frame. For $p=\left(x_{p}, y_{p}\right)$ denoting a pixel on the arena, a difference image $D_{n}(p)$ is found as

$D_{n}(p)=F_{n}(p)-B G(p), \quad 1 \leq n \leq N, \quad \forall p$.

In order to remove the noise and discriminate the animal from the arena, a threshold $\tau$ is applied to the pixel values in $D_{n}$, to obtain a black and white image $I_{n}$, as

$I_{n}= \begin{cases}1 & \text { if } D_{n}(p)>\tau \\ 0 & \text { if } D_{n}(p)<\tau\end{cases}$

$n=1, \ldots, N, \quad \forall p \in D_{n}$.

In $I_{n}$, black represents the test arena, and white represents the subject. The center of gravity of the white area in frame $n$, which we refer as $C_{n}$, is then considered as the location of the animal to be used in tracking the path.

\subsubsection{Formation of basic features}

To represent the discriminative characteristics of the animal's motion numerically, we calculated two basic features for each pixel, sojourn count (SC) and mean instantaneous speed (MIS). For a particular pixel at location $p$ on the arena, $v_{n}(p)$ denotes the presence of a visit of the animal to that location at frame $n$, such that

$v_{n}(p)= \begin{cases}1 & \text { if } p=C_{n}, \\ 0 & \text { otherwise }\end{cases}$

That is, if the center of weight of the mouse at frame $n, C_{n}$, is on pixel $p$, then there is a visit to that pixel at frame $n$. The sojourn count of a pixel $p$ is then defined as the number of all visits to that pixel through the entire video sequence, that is

$S C(p)=\sum_{n \in N} v_{n}(p)$.

Similarly, for a particular pixel $p_{i}=\left(x_{p_{i}}, y_{p_{i}}\right)$, the mean instantaneous speed is the mean value of the displacement values 

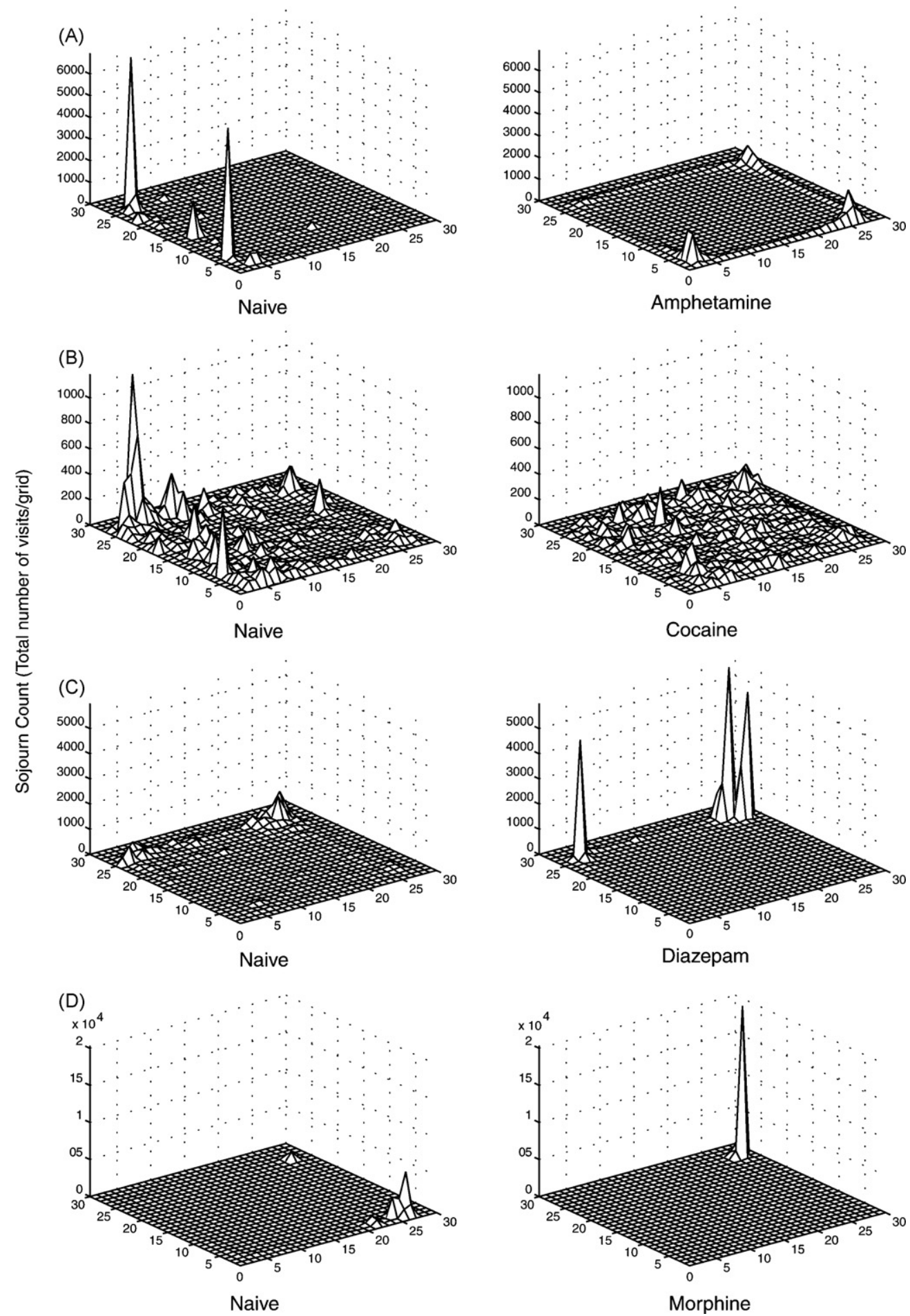

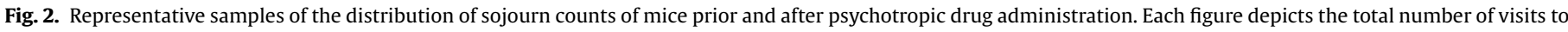

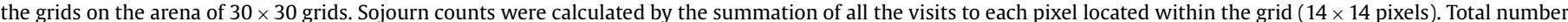

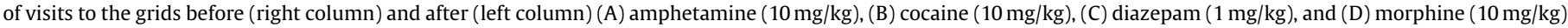
treatment. Each graph displays the results of $40 \mathrm{~min}$ of video recording.

originating from $p_{i}$ at any frame $n$, and moving to $p_{j}=\left(x_{p_{j}}, y_{p_{j}}\right)$ which is the pixel that the center of weight is located on the next frame $n+1$. Thus $\operatorname{MIS}\left(p_{i}\right)$ is calculated as

$$
\operatorname{MIS}\left(p_{i}\right)=\frac{1}{S C\left(p_{i}\right)} \sum_{\substack{n \text { st } \\ C_{n}=p_{i}}} d_{n}\left(p_{i}\right),
$$

where the displacement value $d_{n}\left(p_{i}\right)$ for point $p_{i}$ at a particular frame $n$ is calculated as

$d_{n}\left(p_{i}\right)=\left|\sqrt{\left(x_{p_{i}}-x_{p_{j}}\right)^{2}+\left(y_{p_{i}}-y_{p_{j}}\right)^{2}}\right|, \quad p_{i}=C_{n}, p_{j}=C_{n+1}$.

The arena consists of $M \times M$ pixels, for $M$ being 420 . When SC and MIS are calculated for each pixel, a sparse representation is obtained 

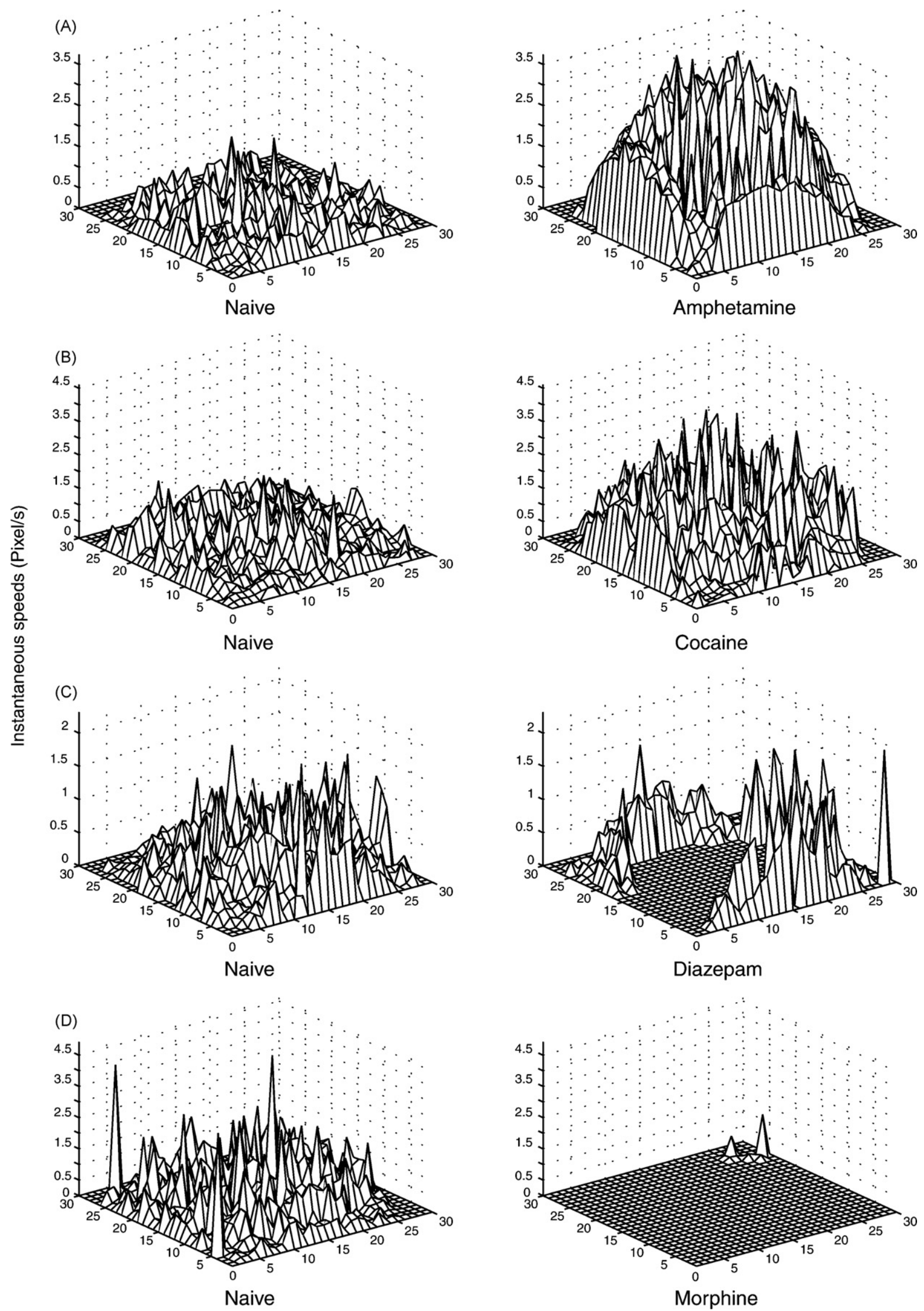

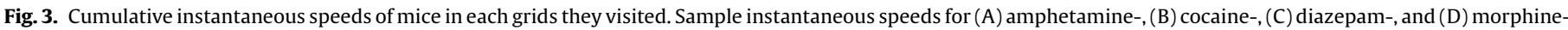

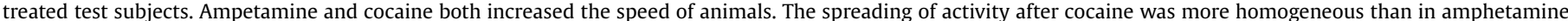

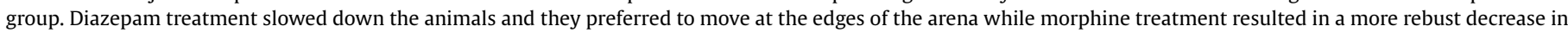
locomotor activity and mice mostly displayed activity at the corners. 
(A)

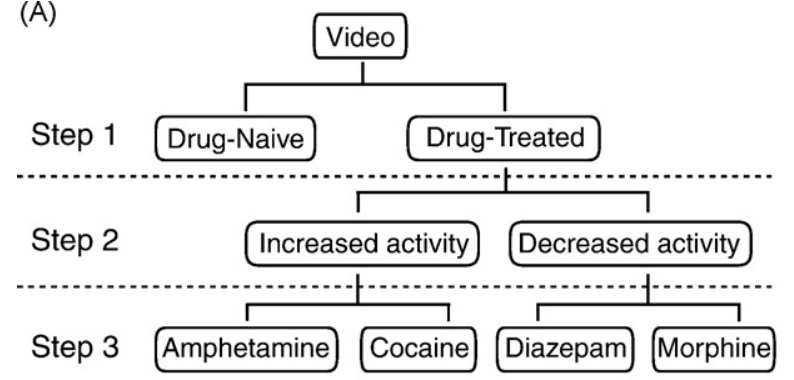

(B)

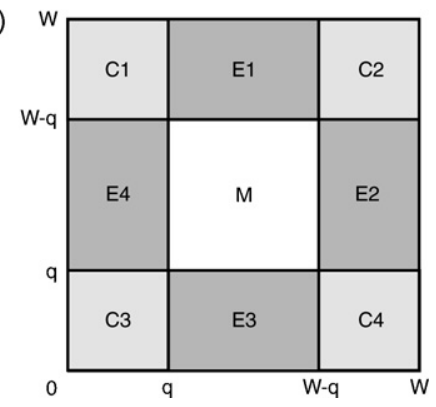

Fig. 4. (A) Steps of Hierarchical Classification algorithm. (B) Locations and borders of three main regions used in the open field experiments.

since it is not very likely that the animal will visit a specific pixel. In order to reduce the noise and to obtain a denser representation, we divided the arena into $W \times W$ grids, each grid containing $w \times w$ pixels, and obtain the features from the groups of pixels in each grid (Fig. 4B).

Let $g_{u v}, 1 \leq u, v \leq W$, be a grid on the arena consisting of $w \times w$ pixels such that

$g_{u v}=\left\{p \mid(u-1) w \leq x_{p}<u w,(v-1) w \leq y_{p}<v w\right\}$.

The sojourn count for a grid $g_{u v}$ is then defined as the sum of the sojourn counts of the pixels in the grid as

$S C\left(g_{u v}\right)=\sum_{\forall p \in g_{u v}} S C(p)$.

The MIS of a grid $g_{u v}$ is defined as the average of the mean instantaneous speed of the pixels for that grid

$\operatorname{MIS}\left(g_{u v}\right)=\frac{1}{S C\left(g_{u v}\right)} \sum_{\forall p \in g_{u v}} \operatorname{MIS}(p)$.

\subsubsection{Formation of complex features}

We observed that under the influence of the psychotropic drugs employed in this study the animals displayed different locomotor activity distribution characteristics. In order to further analyze and differentiate behavioral characteristics we focused on behavioral patterns on local regions of the arena such as corners, edges and centers (Fig. 1B). The corners are represented by $C_{1}, C_{2}, C_{3}$, $C_{4}$, each consisting $q \times q$ grids. Edges are denoted by $E_{1}, E_{2}, E_{3}, E_{4}$ covering $q \times(W-2 q)$. The central region with $(W-2 q) \times(W-2 q)$ grids denoted by $M$. Then we grouped and combined the basic features according to the stated structure. The feature vector for $C_{1}$ in terms of the basic features falling in this region is defined as follows:

$$
\mathbf{S C}\left(C_{1}\right)=\left\lfloor S C\left(g_{11}\right) S C\left(g_{12}\right) \ldots S C\left(g_{1 q}\right) S C\left(g_{21}\right) \ldots S C\left(g_{q q}\right)\right\rfloor .
$$

The aggregate information for the corners and edges are obtained by aligning and adding the corresponding portion of the sojourn count and mean instantaneous speed matrices,

$$
\begin{aligned}
& \mathbf{S C}_{C}=S C\left(C_{1}\right)+S C\left(C_{2}\right)+S C\left(C_{3}\right)+S C\left(C_{4}\right), \\
& \mathbf{M I S}_{C}=\operatorname{MIS}\left(C_{1}\right)+\operatorname{MIS}\left(C_{2}\right)+\operatorname{MIS}\left(C_{3}\right)+\operatorname{MIS}\left(C_{4}\right), \\
& \mathbf{S C}_{E}=S C\left(E_{1}\right)+S C\left(E_{2}\right)^{T}+S C\left(E_{3}\right)^{T}+S C\left(E_{4}\right), \\
& \mathbf{M I S}_{E}=\operatorname{MIS}\left(E_{1}\right)+\operatorname{MIS}\left(E_{2}\right)^{T}+\operatorname{MIS}\left(E_{3}\right)^{T}+\operatorname{MIS}\left(E_{4}\right), \\
& \mathbf{S C}_{M}=S C(M), \\
& \mathbf{M I S}_{M}=\operatorname{MIS}(M),
\end{aligned}
$$

where for each $C_{k}$, the matrices $\operatorname{SC}\left(C_{k}\right)$ and $\operatorname{MIS}\left(C_{k}\right)$ contain the sojourn count and mean instantaneous speed values of the pixels, respectively. $S C\left(E_{k}\right)$ and $\operatorname{MIS}\left(E_{k}\right)$ are defined for $1 \leq k \leq 4$.

\subsubsection{Feature vectors for drug-treated and -untreated} classification of $\mathrm{HC}$

We regarded as a collection of SC and MIS information of $C, E$, and $M$ when the video was labeled as drug-treated or untreated. The activity in regions $C, E$, and $M$ using the mean and standard deviation of sojourn counts and mean instantaneous speeds are:

$$
\begin{aligned}
& \boldsymbol{v}^{I}(C)=\left[\mu\left(\mathbf{S C}_{C}\right) \sigma\left(\mathbf{S C}_{C}\right) \mu\left(\mathbf{M I S}_{C}\right) \sigma\left(\mathbf{M I S}_{C}\right)\right], \\
& \boldsymbol{v}^{I}(E)=\left[\mu\left(\mathbf{S C}_{E}\right) \sigma\left(\mathbf{S C}_{E}\right) \mu\left(\mathbf{M I S}_{E}\right) \sigma\left(\mathbf{M I S}_{E}\right)\right], \\
& \boldsymbol{v}^{I}(M)=\left[\mu\left(\mathbf{S C}_{M}\right) \sigma\left(\mathbf{S C}_{M}\right) \mu\left(\mathbf{M I S}_{M}\right) \sigma\left(\mathbf{M I S}_{M}\right)\right] \text {, }
\end{aligned}
$$

where functions $\mu(\cdot) \sigma(\cdot)$ give the mean and standard deviation. The collection of these three vectors, $\mathbf{V}^{I}$, composed the feature vector of a particular video for classification Step 1,

$\mathbf{V}^{I}=\left\lfloor\mathbf{v}^{I}(C) \mathbf{v}^{I}(E) \mathbf{v}^{I}(M)\right\rfloor$.

\subsubsection{Feature vectors for increased-decreased activity classification of $\mathrm{HC}$}

In this step, absolute changes in the behavioral patterns were used and new features were constructed to display behavioral differences between naïve and drug-treated animals. The feature vectors for naïve and drug-treated recordings at the first level of classification were labeled as $\mathbf{V}_{N}^{I}$ and $\mathbf{V}_{T}^{I}$. Then, the difference of vectors was constructed as

$$
\mathbf{V}^{I I}=\mathbf{V}_{T}^{I}-\mathbf{V}_{N}^{I},
$$

and checked whether $\mathbf{V}^{I I}$ exhibits an increased or decreased activity. $\mathbf{V}^{I I}$ is labeled as $\mathbf{V}_{E}^{I I}$ or $\mathbf{V}_{I}^{I I}$, depending on whether the detection is an increase or a decrease in activity.

\subsubsection{Feature vectors for drug determination step of HC:}

amphetamine-cocaine and morphine-diazepam classifications

The videos labeled as $\mathbf{V}_{E}^{I I}$ were further labeled as amphetamineor cocaine-treated so that the exact drug tag will be determined. Following Step 2 classifier labeling $\mathbf{V}^{I I}$ as $\mathbf{V}_{E}^{I I}$, the Step 3 classifier decided whether the animal is morphine-or diazepam-treated. Since morphine and diazepam both inhibited locomotor activity, only a small part of the arena provided behavioral information. Feature vectors were changed to display activity around the center. If the maximum of the sojourn counts appears at the grid $g_{u^{*} v^{*}}$, where $1 \leq u^{*}, v^{*} \leq W$, we focused on an $\eta \times \eta$ sub-arena around grid $g_{u^{*} v^{*}}$ on the sojourn count and mean instantaneous speed matrices. The sub-arena, denoted by $r^{*}$, is the set of grids

$r^{*}=\left\{g_{u v}: u^{*}-\frac{\eta}{2} \leq u \leq u^{*}+\frac{\eta}{2}-1, v^{*}-\frac{\eta}{2} \leq v \leq v^{*}+\frac{\eta}{2}-1\right\}$.

The sub-arena $r^{*}$ is divided into 9 sub-regions $r_{i j}^{*}$ of equal size where $1 \leq j \leq 3$. The sojourn count $S C\left(r_{i j}^{*}\right)$ for a particular sub-arena $r_{i j}^{*}$ is defined to be the sum of the sojourn counts within that sub-region. 
The mean instantaneous speed value $\operatorname{MIS}\left(r_{i j}^{*}\right)$ of a particular subarena $r_{i j}^{*}$ is defined similarly to be the average of mean instantaneous speed values of the pixels falling into that sub-region.

Similarly, the corners, edges, and center regions, $r_{C}^{*}, r_{E}^{*}, r_{M}^{*}$ are formed by grouping the sub-regions. The sojourn counts and mean instantaneous speeds are calculated by adding the corresponding portions of sojourn count and mean instantaneous speed matrices as:

$$
\begin{aligned}
& \mathbf{S C}_{r_{C}^{*}}=S C\left(r_{11}^{*}\right)+S C\left(r_{13}^{*}\right)+S C\left(r_{31}^{*}\right)+S C\left(r_{33}^{*}\right), \\
& \mathbf{M I S}_{r_{C}^{*}}=\operatorname{MIS}\left(r_{11}^{*}\right)+\operatorname{MIS}\left(r_{13}^{*}\right)+\operatorname{MIS}\left(r_{31}^{*}\right)+\operatorname{MIS}\left(r_{33}^{*}\right), \\
& \mathbf{S C}_{r_{E}^{*}}=S C\left(r_{12}^{*}\right)+S C\left(r_{21}^{*}\right)+S C\left(r_{23}^{*}\right)+S C\left(r_{32}^{*}\right), \\
& \mathbf{M I S}_{r_{E}^{*}}=M I S\left(r_{12}^{*}\right)+M I S\left(r_{21}^{*}\right)+M I S\left(r_{23}^{*}\right)+M I S\left(r_{32}^{*}\right), \\
& \mathbf{S C}_{r_{M}^{*}}=S C\left(r_{22}^{*}\right), \\
& \mathbf{M I S}_{r_{M}^{*}}=\operatorname{MIS}\left(r_{22}^{*}\right) .
\end{aligned}
$$

The feature vector for the corner part is given by

$$
\mathbf{V}_{r_{C}^{*}}=\left\lfloor\mu\left(S C_{r_{C}^{*}}\right) \mu\left(S C_{r_{C}^{*}}\right) \mu\left(M I S_{r_{C}^{*}}\right) \mu\left(M I S_{r_{C}^{*}}\right)\right\rfloor .
$$

The feature vector for the third classification step $\mathbf{V}^{I I I}$ is the concatenation of the feature vectors for all parts, i.e., $r_{C}^{*}, r_{E}^{*}$, and $r_{M}^{*}$.

$\mathbf{V}^{I I I}=\left\lfloor\begin{array}{lll}v_{r_{C}^{*}} & v_{r_{E}^{*}} & v_{r_{M}^{*}}^{*}\end{array}\right.$.

The classifier processes $\mathbf{V}^{I I I}$ labeled it as morphine- or diazepamtreated.

\subsection{Classifiers and validation scheme}

In the classification step, Support Vector Classifier (SVC) and Linear Discriminant Classifier (LDC) are used. SVC is based on support vector machines. Among all hyperplanes, that separate the given classes, there exists a unique hyperplane which gives the maximum margin of separation implying that the distances from the hyperplane to the nearest data points in the separated classes are maximized (Scholkopf et al., 1999). Support vectors are employed in finding this particular hyperplane, making margin of separation maximum (Hearst et al., 1998). The application is implemented in MATLAB (Mathworks, USA) using Pattern Recognition Toolbox PRTools (Duin, 2006). LDC employs linear discriminant functions and looks for a function that gives the most efficient direction for discrimination, namely linear discriminant function (Balakrishnama and Ganapathiraju, 2001).

For all classifiers, we investigated the test and training performance with series of classification experiments. Training performance is described by how well the classifier learns the characteristics of the classes. While exploring training performance, we trained the classifier with a number of training examples and then tested it with exactly the same set of training patterns. The size of training set was increased gradually and the evolution of classification performance against training set size was investigated. Thus it was inferred whether a classifier is able to apprehend the class properties or not. Test performance shows how well the classifier performs when new patterns are investigated for class membership. While measuring test performance, the classifier was trained with a number of training patterns and then tested by new patterns. The number of training patterns was increased step by step and the classifier was tested by the rest of the dataset at each step. As the number of training examples was increased, the classification performance is expected to increase and reach to a steady state value.

In $\mathrm{HC}$ we started from two training samples and increased them until the steady state value of the success rate was reached. LeaveOne-Out (LOO) classification scheme on HC was also used. LOO uses a single observation from the original sample as the validation data and the remaining observations are regarded as the training
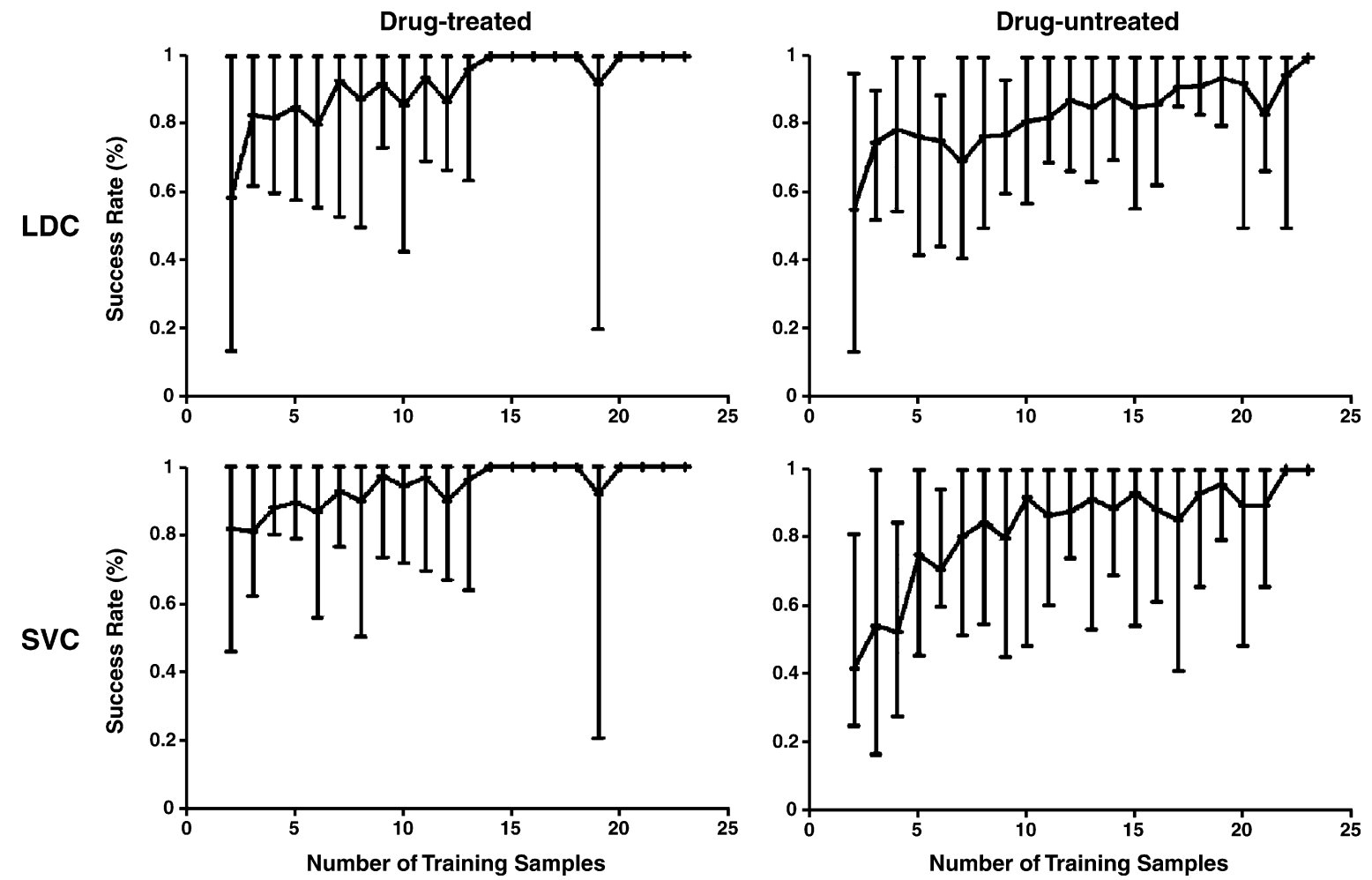

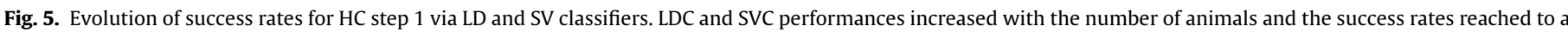
steady value after 10 samples. Data expressed as the mean \pm SD. HC: Hierarchical Classification, SVC: Support Vector Classifier, LDC: Linear Discriminant Classifier. 
Table 1

Success rates for each step of Hierarchical Classification using SV and LD classifiers.

\begin{tabular}{llrrl}
\hline & & SVC & LDC & \# of recordings \\
\hline Step 1 & U & $96 \%$ & $92 \%$ & 24 \\
& T & $100 \%$ & $100 \%$ & 24 \\
Step 2 & I & $92 \%$ & $83 \%$ & 12 \\
& D & $92 \%$ & $92 \%$ & 12 \\
Step 3 & MO & $83 \%$ & $67 \%$ & 6 \\
& DI & $83 \%$ & $67 \%$ & 6 \\
& AM & $67 \%$ & $50 \%$ & 6 \\
& CO & $83 \%$ & $83 \%$ & 6
\end{tabular}

SVC: Support Vector Classifier, U: drug-untreated video recordings, T: drug-treated video recordings, I: increased locomotor activity, MO: morphine, DI: diazepam, AM: amphetamine, $\mathrm{CO}$ : cocaine.

data. This procedure was repeated such that each observation in the sample was used once as the validation data (Duda et al., 2001).

\subsection{Performance}

Success rates of SVC and LDC for HC and LOO are given in Fig. 5 and Table 1. For HC, 4 trials for each number of training samples were made and the average as the success rate was obtained for each number of samples. Success rate of about $90 \%$ was achieved as the number of training samples reached to 10 .

By using the LOO classification scheme we checked whether SVC and LDC mislabeled the feature vectors. Some of the animals in a given set of drug treatment groups displayed nonhomogeneous behavior characteristics, which lead to mislabeling as shown in Table 1.

\section{Discussion}

This paper describes a novel approach to automatically discriminate psychotropic drugs by means of a computerized video-tracking system which accomplishes this process by analyzing the locomotor behavior alterations. This system extracts parameters like sojourn counts, instantaneous speeds, and regional activity from the video recordings and employs a classification algorithm and finally reaches to a conclusion about the drug administered.

Although motion tracking-based computer analysis for behavioral responses has been used for years, the previous approaches were not intended to discriminate a particular drug among other psychoactive agents employed. Automation of the analysis of locomotor activity renders drug screening and behavioral phenotyping of experimental animal studies much easier and faster, consequently this will increase the experimental throughput.

In this study, the algorithm we proposed was based on the analysis of unique feature vectors which were derived from locomotion data. Feature vectors were used to distinguish animal behavior under the influence of a particular psychotropic drug and compare them with the behavior of the animal before and after drug administration and also with other drugs used in this study. These feature vectors constructed by the use of the instantaneous speeds and sojourn counts, adequately represented the drug-induced alterations in behavior and provided the categorization of the animals by feeding the information to the SVC and LDC. Similarly, another method used by Drai et al. (2000) and Kafkafi and Elmer (2005) successfully derived discriminative properties of amphetamine-phencyclidine and amphetamine-cocaine, respectively. This algorithm was based on defining distinct modes of rat locomotion by segmenting the behavioral data as "staying in place" and "going between places" according to the maximum speed attained within the segment (Drai et al., 2001). In the algorithm we proposed, the total number of visits to each pixel were deter- mined and then the cumulative speeds of the animal within these pixels were calculated. Therefore, in our study instead of describing the behavior by segmenting the time series of locomotion data, we used feature vectors that are based on behavior of the animal in a two-dimensional matrix representing the arena.

The feature vectors and classifiers provided a final conclusion of HC with $70-80 \%$ accuracy. Although, the baseline activity of mice displayed variations among the groups, we observed over $96 \%$ correct labeling for the drug-untreated "naïve" animals. The algorithm displayed $92 \%$ accuracy during the analysis step where the drug-treated animals were sorted according to their increased or decreased activities. These findings further support the efficacy of our feature vectors. Additionally, the algorithm was able to match the animals and the drugs administered correctly even under the circumstances where both of the drugs yielded similar cumulative distance curves. For example, both cocaine and amphetamine significantly increased the cumulative distances travelled. In this case, the algorithm achieved $70 \%$ success rate in drug-animal matching. Similarly, diazepam and morphine decreased the cumulative distances travelled, while the success rate was still around $80 \%$. It also should be noted that SVC performed better during drug-animal matching steps according to the LDC.

In the training phase of $\mathrm{HC}$, we observed that the classifiers reliably and quickly learned the characteristics of each classes. Success rates improved as the number of training samples increased. For the number of training samples larger than 10 , the classifiers seemed to fully comprehend the class characteristics. Therefore, in this study 24 mice per group yielded enough discrimination power for the classifiers. The mislabeling of SVC and LDC usually corresponded to the same animals. This finding indicates that these mislabeled animals displayed a different behavior than the rest of the set. We observed that except amphetamine-cocaine classification SVC performed better than LDC in all the classification steps of LOO scheme.

In order to simplify the developmental process of the software we focused on four psychotropic drugs with well-defined behavioral properties. This simplification enabled us to achieve our goal of efficient automatic categorization. However, at this current developmental stage, performance of our software is expected to be lower with drugs from other psychotropic groups and in different experimental designs. For instance, psychoactive drugs with mixed action might yield lower success rates. But at this stage, we did not try to evaluate the effects of psychoactive compounds with mixed behavioral properties, since this was beyond the scope of our study. Although our data was acquired in a paired fashion we also compared the unpaired data with their unmatched controls. In this setting, our software achieved similar success rates with paired design during the comparisons of naïve vs. naïve or naïve vs. drugs inducing behavioral inhibition. However, our software was less accurate when discriminating the psychotropic drugs which induced increased locomotor activity. At this stage, our software utilized only two parameters for the discrimination processes. Addition of more parameters like revisits to recently visited sites and rearing behavior is expected to improve the success rates by increasing the discriminating power. Additionally, our software is a learning-based program; therefore, by introducing additional data acquired either from the drugs we employed and the other psychotropic drugs, improvement of the software performance is expected. Thus, we are planning to implement these modifications into the program and feed with additional data which will possibly increase success rates and reduce the number of possibilities in the case of a new drug or unpaired settings.

In conclusion, the method we developed automatically discriminated drug-treated and -untreated mice and matched the animals with their corresponding psychotropic agents. The feature vectors and classifiers used in our study proved to be effective and sensitive 
enough to represent the behavioral characteristics of the animals under the influence of psychotropic drugs.

\section{Acknowledgement}

This research was funded by Scientific and Technical Research Council of Turkey (TUBITAK, Grant BTT-105E065 and Career 104E065).

\section{References}

Balakrishnama S, Ganapathiraju A, 2001. Linear discriminant analysis: A brief tutorial, institute for signal and information processing. Mississippi State University. Available from: http://www.zemris.fer.hr/predmeti/kdisc/bojana/TutorialLDABalakrishnama.pdf.

Beninger RJ, Cooper TA, Mazurski EJ. Automating the measurement of locomotor activity. Neurobehav Toxicol Teratol 1985;7:79-85

Buresova O, Bolhuis JJ, Bures J. Differential effects of cholinergic blockade on performance of rats in the water tank navigation task and in a radial water maze. Behav Neurosci 1986;100:476-82.

Clarke RL, Smith RF, Justesen DR. An infrared device for detecting locomotor activity. Behav Res Methods Instrum Comput 1985;17:519-25.

Dielenberg RA, Halasz P, Day TA. A method for tracking rats in a complex and completely dark environment using computerized video analysis. J Neurosci Methods 2006;158:279-86.

Drai D, Benjamini Y, Golani I. Statistical discrimination of natural modes of motion in rat exploratory behavior. J Neurosci Methods 2000;96:119-31.

Drai D, Kafkafi N, Benjamini Y, Elmer G, Golani I. Rats and mice share common ethologically relevant parameters of exploratory behavior. Behav Brain Res 2001;125:133-40.
Duda RO, Hart PE, Stork DG. Pattern classification. New York: Wiley; 2001

Duin RPW. PRTools Version 4.0, A Matlab Toolbox for Pattern Recognition. Netherlands: Pattern Recognition Group, Delft University; 2006.

Hearst MA, Dumais ST, Osman E, Platt J, Scholkopf B. Support vector machines intelligent systems and their applications. IEEE 1998;13(July-August(4)): $18-28$.

Kafkafi N, Elmer GI. Activity density in the open field: a measure for differentiating the effect of psychostimulants. Pharmacol Biochem Behav 2005;80: 239-49.

Noldus LP, Spink AJ, Tegelenbosch RA. EthoVision: a versatile video tracking system for automation of behavioral experiments. Behav Res Methods Instrum Comput 2001;33:398-414.

Pasquali V, Renzi P. On the use of microwave radar devices in chronobiology studies: an application with Periplaneta americana. Behav Res Methods 2005;37: 522-7.

Scholkopf B, Burges CA, Smola J. Advances in kernel methods: Support vector learning. Cambridge, MA: MIT Press; 1999.

Robles E. A method to analyze the spatial distribution of behavior. Behav Res Methods Instrum Comput 1990;22:540-9.

Shih YH, Young MS. Integrated digital image and accelerometer measurements of rat locomotor and vibratory behaviour. J Neurosci Methods 2007;166:81-8.

Spruijt BM, Gispen WH. Prolonged animal observation by use of digitized videodisplays. Pharmacol Biochem Behav 1983;19:765-9.

Spruijt BM, Buma MOS, van Lochem PBA, Rousseau JBI. Automatic behavior recognition: what do we want to recognize and how do we measure it? In: Proceedings of 2nd international conference on methods and techniques in behavioral research measuring behavior; 1998. p. 264-6.

Teicher MH, Andersen SL, Wallace P, Klein DA, Hostetter J. Development of an affordable high-resolution activity monitor system for laboratory animals. Pharmacol Biochem Behav 1996;54:479-83.

Vorhees CV, Acuff-Smith KD, Minck DR, Butcher RE. A method for measuring locomotor behavior in rodents: contrast-sensitive computer-controlled video tracking activity assessment in rats. Neurotoxicol Teratol 1992;14:43-9. 